\title{
Study of dimorphic anemia in adults with reference to basic etiology
}

\author{
Deepthi. A ${ }^{1}$, CSBR Prasad ${ }^{2}$, Raghavendra Prasad B. N. ${ }^{3}$ \\ ${ }^{1}$ Assistant Professor, Dept. of Pathology, Shridevi Medical College, Karnataka, ${ }^{2,3}$ Professor, ${ }^{2}$ Dept. of Pathology, \\ ${ }^{3}$ Dept. of Medicine, ${ }^{2,3}$ Sri Devaraj URS Medical College, Kolar, Karnataka, India
}

*Corresponding Author:

Email: drdeepthia@gmail.com

\begin{abstract}
Objective: To investigate the basic cause of dimorphic anemia in adult patients using hematological parameters, bone marrow iron stores, serum vitamin B12 and folate assays.

Materials and Methods: Hematological parameters and bone marrow, serum vitamin B12 and folate assays of 62 patients with dimorphic anemia were studied to evaluate the basic etiology in dimorphic anemia.

Results: The mean MCV was $95.01 \pm 13.14 \mathrm{fl}$ and MCV was normal in $56.45 \%$ cases. BM examination showed megaloblastic maturation in $32.36 \%$, megaloblastic with micronormoblastic maturation in $64.52 \%$ cases. BM iron stores were deficient in $32.26 \%$ cases. $43.55 \%$ cases had only folic acid deficiency, $19.35 \%$ cases had only B12 deficiency and $27.42 \%$ cases had both B12 and folate deficiency. Combined iron and vitamin deficiencies in $25.8 \%$ cases. The mean MCV, MCH, MCHC and RDW were significantly high in combined deficiencies.

Conclusion: The common basic cause for dimorphic anemia was folate deficiency. MCV is unreliable as a screening parameter for anemia with vitaminB12 or folate deficiencies. Concomitant deficiencies of B12 or folate with iron deficiency are not infrequent; one type of anemia may mask the other. Serum assays will be appropriate to establish the cause in cases with concomitant deficiencies, as it may result in discordant results with various other tests like hematological parameters.
\end{abstract}

Keywords: Anemia, Dimorphic, Folate, Megaloblastic, Myelodysplasia, Vitamin B12.

Received: $24^{\text {th }}$ May, 2017

\section{Introduction}

The most common hematological disorder in all age groups is anemia. Globally, anemia affects 1.62 billion people, which corresponds to $24.8 \%$ of the population. ${ }^{1}$ Dimorphic anemia has a complex pathogenesis with involvement of more than one deficiency state, usually due to deficiency of both iron and vitamin B12 or folic acid. $^{2}$ This condition may be reflected by increased RDW in the presence of normal MCV, with dimorphic blood picture showing two RBC populations, that is combination microcytic hypochromic and macrocytic normochromic cells. ${ }^{3}$ Myelodysplasia may be an underlying pathology in older age group, as the dimorphic anemia is characteristic of sideroblastic anemia. ${ }^{4}$ Concomitant vitamin B12 or folate deficiency with iron deficiency results in masked megaloblastosis, where the vitamin deficiencies are not associated with classic findings of megaloblastic anemia. ${ }^{2}$ If treated with iron alone megaloblatosis will be unmasked. ${ }^{5}$

Our study signifies the importance of early diagnosis of specific vitamin deficiencies and thereby preventing the further life threatening consequences.

\section{Materials and Methods}

The study included 62 adult patients with mean corpuscular volume $>70 \mathrm{fl}$, RDW $>15 \%$, RBC histogram with double peaks and peripheral blood smear showing dimorphic blood picture. Pregnant females, cases with increased reticulocyte count and who had received blood transfusions and specific therapy were excluded. The basic etiology in dimorphic anemia is evaluated using bone marrow iron stores, serum vitamin B12 and folate assays and compared with hematological parameters. The study had been approved by Institutional ethics committee.

Bone marrow (BM) aspiration was performed on these patients at the posterior superior iliac spine. Smears were prepared, airdried and stained with Giemsa and perls' stain. Giemsa stained smears were looked for cellularity, erythroid hyperplasia, maturation and dysplasia of erythroid, myeloid and megakaryocytic series. Perls' stained smears were looked for iron stores and graded according to Gale's method.

Blood sample was collected in plain vacutainers, serum was separated immediately. Serum levels of folic acid and vitamin B12 had been carried out using proper controls. Vitamin B12 deficiency was defined as serum levels of 
B12 $\leq 211 \mathrm{pg} / \mathrm{ml}$. Folate deficiency was defined as serum levels of folic acid $\leq 5.3 \mathrm{ng} / \mathrm{ml}$.

\section{Statistical Analysis}

Percentage of each cause was calculated. The mean and standard deviation were used for statistical analysis. ANOVA test was used to test the variation of mean of hematological parameters in various groups and its significance. $\mathrm{P}<0.05$ was considered statistically significant.

\section{Results}

The age group of our cases ranged from 20 to 75 years with mean age of $40.95 \pm 17.61$ years. Majority of patients $23(37.1 \%)$ belonged to second decade, followed by fifth decade constituting $14(22.58 \%)$ cases. Male: Female ratio was $1.1: 1$. 33(53.2\%) were male and 29(46.8\%) were female.

The commonest presenting complaint was fatiguability in $37(59.68 \%)$ cases, followed by fever in $31(50 \%)$ cases, $15(24.19 \%)$ cases presented with respiratory distress, 6(9.68\%) patients had associated bleeding manifestations like epistaxis and bleeding per rectum. Other symptoms like headache, chest pain, pain abdomen, joint pain and diarrhea were seen in 18 (29\%) cases. Pallor was present in all patients. Icterus was seen in $11.29 \%$ (7/62). Hepatomegaly and splenomegaly was seen in $16.13 \%$ and $12.9 \%$ of patients respectively.

The hemoglobin concentration ranged from $1.49 \mathrm{gm} / \mathrm{dl}$ to $10.5 \mathrm{gm} / \mathrm{dl}$ with the mean of $5.74 \pm 2.43 \mathrm{gm} / \mathrm{dl}$. Severe anemia with $\mathrm{Hb}<8$ $\mathrm{gm} / \mathrm{dl}$ was seen in $49(79.03 \%)$ cases, moderate $(8-10 \mathrm{gm} / \mathrm{dl})$ in $12(19.35 \%)$ cases and mild(> 10 $\mathrm{gm} / \mathrm{dl})$ in only one $(1.61 \%) .22$ cases had only dimorphic anemia, 19 cases had bicytopenia, either dimorphic anemia with leucopenia or thrombocytopenia and 21 cases had pancytopenia (dimorphic anemia with leucopenia and thrombocytopenia) (Table 1)

Table 1: Distribution of cases based on cytopenia

\begin{tabular}{|l|c|c|}
\hline \multicolumn{1}{|c|}{ Cytopenia } & Number of patients & Percentage (\%) \\
\hline Only dimorphic anemia & 22 & 35.48 \\
\hline Bicytopenia & 19 & 30.65 \\
\hline Pancytopenia & 21 & 33.87 \\
\hline Total & 62 & 100 \\
\hline
\end{tabular}

Mean corpuscular volume (MCV) ranged from 80 to $120 \mathrm{fl}$ with mean MCV of $95.01 \pm 13.14 \mathrm{fl}$. MCV was <97 fl in $35(56.45 \%)$ patients and high MCV was seen in 27 (43.55\%) patients. Among the patients with high MCV, $63 \%$ were male and $37 \%$ were female. Mean corpuscular hemoglobin( $\mathrm{MCH})$ ranged from 16.2 to $44.8 \mathrm{pg}$ with mean $\mathrm{MCH}$ of $29.31 \pm 8 \mathrm{pg}$. Mean corpuscular hemoglobin concentration(MCHC) ranged from 25.4 to $40.5 \mathrm{gm} / \mathrm{dl}$ with mean MCHC of $32.22 \pm 2.85 \mathrm{gm} / \mathrm{dl}$. The RDW ranged from 15.1 to $25.9 \%$, with mean RDW of $17 \pm 2.61 \%$

Bone marrow examination showed normocellular marrow in 20(32.36\%) cases and hypercellular marrow in $42(67.74 \%)$ cases. Erythroid hyperplasia was seen in $58(93.54 \%)$ cases, which is indicative of bone marrow response to anemia. Total megaloblastic maturation was seen in $20(32.36 \%)$ and $40(64.52 \%)$ patients showed both megaloblastic and micronormoblastic maturation (Chart 1). $31(50 \%)$ patients showed dyserythropoiesis, all of these cases also showed giant metamyelocytes and $5(8 \%)$ patients showed only giant metamyelocytes without dyserythropoiesis.

\section{Chart 1: Distribution of cases based on Erythroid maturation and BM diagnosis}

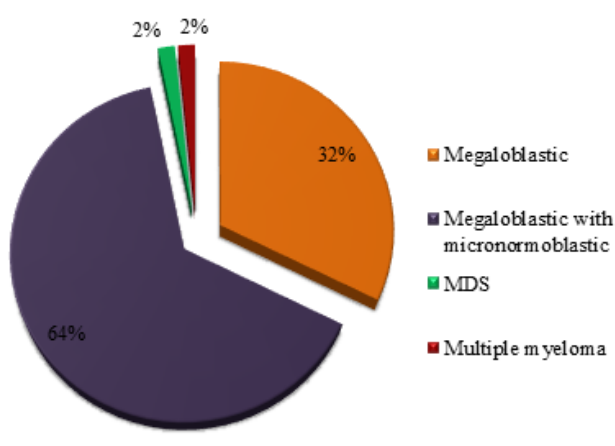


A 40 year old male patient presented with fever and pancytopenia with $\mathrm{Hb}$ of $7.3 \mathrm{gm} / \mathrm{dl}$, bone marrow showed features of myelodysplastic syndrome (MDS), with erythroid hyperplasia showing dyserythropoiesis, dysmyelopoiesis with $9 \%$ of blasts. The iron stores were increased. Another case, 65 year old female presented with pathological fracture with $\mathrm{Hb}$ of $7 \mathrm{gm} / \mathrm{dl}$ and dimorphic anemia on peripheral blood smear examination. BM showed normoblastic erythroid maturation with features of multiple myeloma (MM), having $40 \%$ plasmablasts.

The mean of MCV and $\mathrm{MCH}$ were high in megaloblastic maturation, as compared with patients with both micronormoblastic and megaloblastic maturation. But, was not statistically significant (Chart 2).

Chart 2: Mean of RBC indices among different groups based on Bone marrow diagnosis

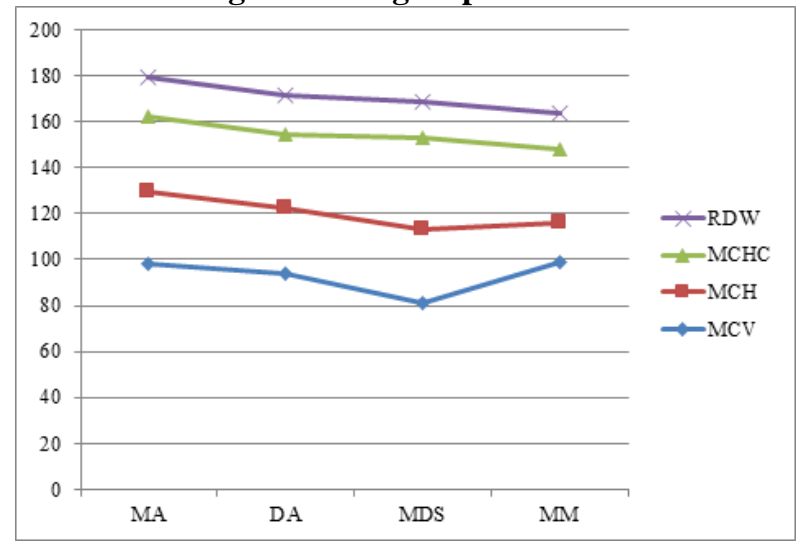

MA - Megaloblastic anemia, DA - Dimorphic anemia, MDS - Myelodysplatic syndrome, MM Mutiple myeloma.

In our study, $40 \%$ of patients with megaloblastic maturation presented with pancytopenia. $30 \%$ of patients with both megaloblastic and micronormoblastic maturation presented with pancytopenia. In patients with megaloblastic maturation, $3(15 \%)$ cases showed normocellularity and $17(85 \%)$ cases showed hypercellularity. In patients with dimorphic maturation, $16 \quad(40 \%)$ cases showed normocellularity and $24(60 \%)$ cases showed hypercellular bone marrow.

Bone marrow iron stores were assessed on Perls' stained bone marrow aspirate smears of our cases by Gale's method. Bone marrow iron grade ranged from grade 0 to grade 5 and was categorized as deficient, normal and increased stores. Deficient iron stores was seen in 20 $(32.26 \%)$ cases, normal stores in 35 (56.45\%) cases and increased stores in $7(11.29 \%)$ cases.

On comparison of hematological parameters between cases with adequate iron stores and deficient stores, the mean of $\mathrm{MCH}$ and $\mathrm{MCHC}$ were low in cases with deficient iron stores and were statistically significant, with $\mathrm{p}$ - value < 0.05 , obtained by one - way ANOVA.

In our study, $27(43.55 \%)$ cases showed only folic acid deficiency. 12(19.35\%) cases showed only vitamin B12 deficiency. $17(27.42 \%)$ cases showed both vitamin B12 and folic acid deficiency. $6(9.67 \%)$ cases showed normal levels of serum vitamin B12 and folic acid.

All cases of megaloblastic anemia showing deficient iron stores (IDA) are associated with vitamin deficiency (either vitamin B12 or folic acid), 10 (50\%) patients showed only serum folic acid deficiency, 6(30\%) patients showed only vitamin B12 deficiency and $4(20 \%)$ patients showed both vitamin $\mathrm{B} 12$ and folic acid deficiency. Among patients with both micronormoblastic and megaloblastic maturation, $17(42.5 \%)$ cases were associated with deficient iron stores (IDA), of which only $14(35 \%)$ cases showed mixed deficiency of both iron and vitamins, $17(42.5 \%)$ patients showed only folic acid deficiency, 6 (15\%) patients showed only vitamin B12 deficiency and 13 (32.5\%) patients showed both vitamin B12 and folic acid deficiency (Table 2). In our study, $25.8 \%$ cases were associated with both iron and vitamin B12 and / folate deficiency (mixed deficiency). 
Table 2: Distribution of cases with Iron deficiency anemia, vitamin B12 and folic acid deficiency in megaloblastic anemia and dimorphic anemia (micronormoblastic and megaloblastic)

\begin{tabular}{|l|c|c|}
\hline \multicolumn{1}{|c|}{ Deficiencies } & Megaloblastic $(\mathbf{n = 2 0})$ & Dimorphic $(\mathbf{n = 4 0})$ \\
\hline IDA & 2 & 17 \\
\hline Vitamin B12 & 6 & 6 \\
\hline Folic acid & 10 & 17 \\
\hline $\begin{array}{l}\text { Vitamin B12 \& Folic } \\
\text { acid }\end{array}$ & 4 & 13 \\
\hline Vitamins \& IDA & 2 & 14 \\
\hline
\end{tabular}

The mean $\mathrm{MCV}, \mathrm{MCH}, \mathrm{MCHC}$ and $\mathrm{RDW}$ were high in combined (vitamin B12 and folic acid) deficiency compared to all other groups. $\mathrm{MCV}$ and $\mathrm{MCH}$ were statistically significant between IDA cases and combined (vitamin B12 and folic acid) deficiency with $\mathrm{p}$ value of $<0.05$ and $<0.01$ respectively, obtained by ANOVA.

\section{Discussion}

Anemia is the most common treatable problem in developing countries. ${ }^{6}$ Nutritional deficiencies is the most common cause of anemia, in country like India. Good proportion of cases show combined deficiency of iron, vitamin B12 and folic acid, where multiple factors affect the diagnostic parameters, resulting in discordant results of tests like bone marrow morphology, iron stores and iron studies. ${ }^{7}$ Iron deficiency anemia is the first common nutritional anemia in our country, followed by megaloblastic anemia. People with low socioeconomic group are the ones who are mainly affected.

Majority of cases in this study were in second decade of life. There was no clear male preponderance seen. In this study, the commonest presenting complaint was fatiguability in $59.68 \%(37 / 62)$ followed by fever in $50 \%$ (31/62). Tahlan et al also showed the incidence of fever in megaloblastic anemia varying from $28 \%$ to $60 \%$. It may be attributed to leucopenia or premature destruction of hematopoietic precursors and release of intracellular substances functioning as pyrogens. ${ }^{7,8}$

The present study showed, only dimorphic anemia in 22(35.5\%) cases, anemia with leucopenia in $8(12.91 \%)$ cases and thrombocytopenia in $11(17.72 \%)$ cases, pancytopenia in $21(33.87 \%)$ cases, which was similar to study conducted by Khan $\mathrm{S}$ et al. ${ }^{7}$ In our study, MCV was < $97 \mathrm{fl}$ in $35(56.45 \%)$ patients and high MCV was seen in 27 (43.55\%) patients, our finding is consistent with a study where high MCV was seen in $41.7 \%$ patients. $^{7}$ Concomitant iron deficiency and fragmentation of red cells may result in normal MCV. ${ }^{7} \mathrm{RDW}$ is useful in differentiating megaloblastic anemia (high MCV with high RDW) and other macrocytic anemia (high MCV and normal RDW). ${ }^{9}$ Bone marrow examination showed normocellular marrow in $32 \%$ cases and hypercellular marrow in $68 \%$ cases, which is similar to the study by Metikurke SH et al where $36.02 \%$ cases had normocellular marrow and $57.72 \%$ cases had hypercellular marrow. Megaloblastic maturation was seen in $32.36 \%$ patients and megaloblastic with micronormoblastic maturation in $64.52 \%$ of our patients. Megaloblastic bone marrow is hypercellular, with accumulation of primitive cells. Megaloblastic anemia may be associated with normoblastic or micronormoblastic maturation. ${ }^{10}$

Bone marrow iron stores in uncomplicated megaloblastic anemia are normal or increased. Assessment of iron stores in bone marrow is considered as "Gold standard" test. ${ }^{11}$ The Gale's grading revealed normal stores in 56.45\% cases, iron deficient stores in $32.26 \%$ cases and increased stores in $11.29 \%$ cases. Megaloblastic anemia with adequate iron stores and low iron stores was present in $65 \%$ and $10 \%$ respectively, similar to studies by Tahlan et al in $69.1 \%$ and $17 \%$ cases respectively. ${ }^{12}$

DNA synthesis is impaired in Folic acid and vitamin B12 deficiency, resulting in impaired and ineffective erythropoiesis. Vitamin B12 or folate deficiency is associated with elevated homocysteine levels, major risk factor of occlusive vascular diseases. The present study shows isolated folate and vitamin B12 deficiency in $43.5 \%$ and $19.3 \%$ cases respectively. Combined folate and vitamin B12 deficiencies were seen in $27.42 \%$ cases. The present study showed folate deficiency as the major cause of dimorphic anemia. Other studies on various other groups had shown similar results (Table 3). Severe folate deficiency may result in low vitamin B12 levels, which is corrected on monotherapy with folic acid alone. Upto $5 \%$ of patients with megaloblastic anemia may have results within reference range. ${ }^{13}$ 
Table 3: Comparison of percentage of cases with vitamin B12, folate and combined deficiencies in our study with other studies conducted on various other groups

\begin{tabular}{|c|c|c|c|c|}
\hline Studies & Study group & Folate defn & B12 defn & Combined \\
\hline Premkumar et $\mathrm{al}^{6}$ & Adults-pancytopenia & $7.14 \%$ & $81 \%$ & $3.51 \%$ \\
\hline Suarez T et al ${ }^{14}$ & Adolescents & $91.02 \%$ & $19.23 \%$ & -- \\
\hline Bhende et al $^{15}$ & Nutri. MA & $54.7 \%$ & $7 \%$ & $7.3 \%$ \\
\hline Present study & Adults-DA & $43.55 \%$ & $19.35 \%$ & $27.42 \%$ \\
\hline
\end{tabular}

Racial and ethnic factors may influence the normal levels and metabolism of these vitamins. Variation of nutritional deficiencies across the geographical regions is associated with nutritional profile and associated infectious and inflammatory diseases. ${ }^{16}$ The laboratory diagnosis of folate deficiency has been more difficult than that of vitamin B12, as serum folate levels are markedly affected by a short period of dietary deprivation and recent alcohol ingestion. ${ }^{17}$ Dietary deprivation may be reason for more patients with folate deficiency in our study, as $79.03 \%$ patients were severely anemic with severe illness. $50 \%$ of patients presented with fever. Studies have shown that folate store depletion may be consequence of rise in temperature. ${ }^{18}$

Nutritional deficiency may be the reason for more of folate deficiency in majority of our patients. Its postulated that, reduced endogenously available B12 may be a "complication of migration to a more sanitized environment". ${ }^{19}$ In the present study, population studied was from rural areas, and as a result vitamin B12 deficiency may not be as prevalent as shown by other studies conducted at urban areas.

Iron deficiency masks the expression of vitamin B12 and folate deficiency. If the vitamin deficiency predominates, full morphologic expression of megaloblastic hematopoiesis is seen, but response to vitamin will be incomplete. If iron deficiency predominates, morphologic expression of vitamin deficient state is limited to only myeloid series. Thus, it's very important to know the basic etiology and treat efficiently. In the present study $25.8 \%$ of cases showed both iron and vitamin deficiency. In a study by Suarez $\mathrm{T}$ et al on adolescents showed simultaneous iron and folate deficiency in $30.76 \%$ of anemic cases. ${ }^{14}$ As iron status was assessed on bone marrow iron stores, it may not be reliable due to discordant results obtained in cases of masked megaloblastosis due to associated iron deficiency anemia. In therapeutic point of view, accurate identification of iron, vitamin B12 and folic acid deficiencies are important.

Folate supplementation in the presence of occult vitamin B12 deficiency corrects the hematological signs but neurological symptoms remain unaltered or may get precipitated. ${ }^{20}$ When iron therapy is given to the patient with both megaloblastic and iron deficiency anemia, the bone marrow findings of megaloblastic anemia is unmasked and symptoms persist. Other than hematological and neurological manifestations, vitamin $\mathrm{B} 12$ and folate deficiencies are less commonly associated with occlusive cardiovascular diseases, osteoporosis and pathological fractures. ${ }^{21}$

Megaloblastic anemia develops over a period of time and most of them are well compensated. Urgent blood transfusion or any form of therapy is not indicated before collection of serum samples for vitamin assays. Assays alone determine which vitamin is deficient. Megaloblastic anemia results in life threatening complications if unrecognized and not treated adequately.

\section{Conclusion}

The common basic cause for dimorphic anemia was folate deficiency. Majority showed normal MCV even with vitamin B12 \& folate deficiency. MCV alone is unreliable as a screening parameter in anemia with vitamin B12 or folate deficiencies, with associated iron deficiency. Concomitant deficiencies of B12 or folate with iron deficiency are not infrequent, constituting $25 \%$ of our cases. Its important to establish the correct diagnosis to avoid inappropriate therapy. Serum assays will be appropriate to establish the cause in concomitant deficiencies, as it may result in discordant results in hematological parameters, BM morphology and iron stores. Thus, hematological parameters alone are likely to miss deficient cases.

\section{References}

1. Benoist B, McLean E, Egli I, Cogswell M, Wojdyla D. Worldwide prevalence of anemia 1993-2005.WHO Global Database on Anemia. Geneva, Switzerland: World Health Organization, 2008; p.1-51.

2. Antony AC. Megaloblastic anemias. In: Hoffman R, Benz EJ, Silberstein LE, Heslop H, Weitz J, Anastasi J editors. Hematology Basic principles and practice. $6^{\text {th }}$ Edition. Churchill Livingstone;2012.p519-56. 
3. Bain B J. Blood cell morphology in health and disease.In: Lewis S M, Bates I, editors. Dacie and Lewis Practical Haematology, $10^{\text {th }}$ Edition.Churchill Livingstone:Elsevier Ltd;2011.p.79-114.

4. Perkins SL. Examination of the blood and bone marrow. In: Greer JP, Foester J, Rodgers GM, editors. Wintrobe's Clinical Hematology. $12^{\text {th }}$ Edition. Philadelphia: Lippincott Williams \& Wilkins;2009:p.1-20.

5. Spivak JL. Masked megaloblastic anemia. Arch Intern Med 1982;142:2111-5

6. Premkumar M, Gupta N, Singh T, Velpandian T. Cobalamin and folic acid status in relation to the etiopathogenesis of pancytopenia in adults at a tertiary care centre in North India. Anemia 2012;2012, Article ID 707402, 12 pages. doi:10.1155/2012/707402.

7. Khan S, Raziq F, Qureshi H. Association of megaloblastic anemia with peripheral cytopenias. J Postgrad Med Inst 2009;23(1):46-50.

8. Manuel K, Padhi S, Varghese RGB. Pyrexia in a patient with megaloblastic anemia: A case report and literature review. Indian J Med Sci 2013;38(2):198-202.

9. Bain BJ, Lewis SM, Bates I. Basic haematological techniques.In: Bain B J, Bates I.editors. Dacie and Lewis Practical Haematology, $10^{\text {th }}$ Edition.Churchill Livingstone: Elsevier Ltd;2011.p11-24

10. Metikurke SH, Rashmi K, Bhavika R. Correlation of Bone marrow aspirate, biopsies and touch imprint findings in pancytopenia. J Hematol 2013 May [cited 2013 June] 2(1):8-13. Available from: http://www.jh.elmerpress.com/index.php/jh/articl e/view/76/53

11. Thomas DW, Hinchliffe RF, Briggs C, Macdougall IC, Littlewood T, Cavill I. Guideline for the laboratory diagnosis of functional iron deficiency. Br J Haematol 2013;161(5):639-48.

12. Tahlan A, Bansal C, Palta A, Chauhan S. Spectrum and analysis of bone marrow findings in anemic cases. Indian J Med Sci 2008;62(8):336-9.

13. Hamilton M, Blackmore S. Investigation of megaloblastic anemia-cobalamin, folate and metabolic status. In: Lewis S M, Bain B J, Bates I,editors.Dacie and Lewis Practical Haematology, $10^{\text {th }}$ Edition.Churchill Livingstone:Elsevier Ltd;2011.p161-86

14. Suarez T, Torrealba M, Villegas N, Osorio C, Garcia-Casal MN. Iron, folic acid and vitamin B12 deficiencies related to anemia in adolescents from a region with a high incidence of congenital malformations in Venezula. Arch Latinoam Nutr 2005;55(2):118-23.

15. Bhende YM. Some experiences with nutritional megaloblastic anemia. J Postgrad Med 1965;11(4):145-55.

16. Bhardwaj A, Kumar D, Raina SK, Bansal P, Bhushan S, Chander V. Rapid assessment for coexistence of vitamin B12 and iron deficiency anemia among adolescent males and females in Northern Himalayan state of India. Anemia 2013;2013:Article ID 959605, 5 pages. doi: $10.1155 / 2013 / 959605$.

17. Frenkel EP, Yardley DA. Clinical and laboratory features and sequelae of deficiency of folic acid(folate) and vitamin B12(cobalamin) in pregnancy and gynecology. Hematol Oncol Clin North Am 2000;14:1079-100.

18. Iqbal SP, Kakepoto GN, Iqbal SP. Vitamin B12 deficiency-a major cause of megaloblastic anemia in patients attending a tertiary care hospital. $\mathbf{J}$ Ayub Med Coll Abbottabad 2009;21(3):92-4.

19. Khandari U, Sharma A. Megaloblastic anemia: prevalence and causative factors. Natl Med J India 2007;20(4):172-5.

20. Singh B, Gupta VK, Venkatesan M. Burden of vitamin B12 deficiency in urban population in Delhi, India: A hospital based study. Int J of Pharma \& Bio Sci 2011;2(1):521-7.

21. Clarke R, Refsum H, Birks J, Evans JG, Johnston $\mathrm{C}$, Sherliker P et al. Screening for vitamin B12 and folate deficiency in older persons. Am J Clin Nutr 2003;77(5):1241-7. 